Pure and Applied Mathematics Quarterly

Volume 6, Number 2

(Special Issue: In honor of

Michael Atiyah and Isadore Singer)

$603-622,2010$

\title{
Approaches to Generalize Contact Structures
}

\author{
Yat Sun Poon and Aïssa Wade
}

\begin{abstract}
After defining the concept of generalized almost contact structures, we examine integrability of such structures from both a Sasakian perspective in terms of a cone construction and from a Courant algebroid perspective.
\end{abstract}

Keywords: Contact, complex, symplectic, generalized complex.

\section{INTRODUCTION}

Contact geometry is a classical subject [15]. It is closely related to complex and symplectic structures through real hypersurface construction or cone construction. It has many facets of generalization in the last fifty years. In particular, there is a notion of almost contact structure and a notion of almost cosymplectic structure.

In recent years, there has been tremendous development in understanding the relation and interplay between complex and symplectic geometry beyond classical Kählerian geometry. In particular, the concept of generalized complex geometry successfully integrates complex and symplectic structures into a single category of geometry [9] [11]. Then a notion of generalized almost contact structures emerges [12]. Its development is based on the theory of Dirac structures and 1-jet bundles of the underlying manifolds.

In this paper, we consider "generalized almost contact structures of codimension1 " in the sense of Vaisman as an alternative [23]. Although this definition of a

Received May 1, 2008. 
generalized almost contact structure is more restrictive than the one given in [12], it has advantage that it is simply formulated in terms of tensorial objects.

We then investigate different perspectives on "integrability" of generalized almost contact structures. One approach is inspired by Sasaki's method of extending a given structure from an odd-dimensional manifold to the cone over it, and then considering integrability of an almost complex structure on even-dimensional manifolds. Another is inspired by Courant and Weinstein's approach to Dirac structures [6] [7].

Historically, there is a distinction between contact structures and the geometry determined by a contact 1-form. As on orientable manifolds these concepts are equivalent [10] (see also [1]), to avoid complication irrelevant to our considerations, throughout this paper all manifolds under considerations are assumed to be connected and oriented. As an initial investigation, we focus on tensorial objects, and postpone consideration of equivalent relations to future.

\section{Classical geometry}

2.1. Contact structures as almost cosymplectic structures. An almost symplectic structure on a $2 n$-dimensional manifold is a 2 -form $\omega$ such that $\omega^{n}$ is non-zero everywhere. As a $G$-structure, the manifold $M$ has a $S p(n, \mathbb{R})$-structure [4] [13]. The integrability of this $G$-structure is equivalent to $d \omega=0$. In such case, we simply address the 2 -form $\omega$ as a symplectic structure or a symplectic form.

On odd-dimensional manifolds, contact structures share many common features with symplectic structures. They are often studied along with symplectic structures in the realm of classical mechanics, beginning with S. Lie [15]. Suppose that $M$ is a $(2 n+1)$-dimensional manifold with a 1 -form $\eta$ such that $\eta \wedge(d \eta)^{n}$ is non-zero everywhere, then the 1-form $\eta$ is a contact structure or a contact 1-form. The kernel of the 1-form ker $\eta$ is called contact distribution. Note that the restriction of the 2 -form $d \eta$ to the associated contact distribution is non-degenerate. Given a contact one-form, there is a unique vector field $F$ such that $\iota_{F} \eta=1$ and $\iota_{F} d \eta=0$. This vector field is known as the Reeb field of the contact form $\eta$. 
When studying infinitesimal automorphisms of symplectic structures, Libermann developed the concept of almost cosymplectic structures [14] As a Gstructure, it is a reduction of the structure group of a $(2 n+1)$-dimensional manifold from $G L(2 n+1, \mathbb{R})$ to $\{1\} \times S p(n, \mathbb{R})[8][14]$. In terms of tensors, it is equivalent to the choice of a 1 -form $\eta$ and a 2 -from $\theta$ such that $\eta \wedge \theta^{n} \neq 0$ at every point of the manifold. An almost cosymplectic structure $(\eta, \theta)$ is a cosymplectic structure if both $\eta$ and $\theta$ are closed [14]. It is immediate that contact forms constitute a subclass of almost cosymplectic structures with $\theta=d \eta$.

2.2. Contact structures as almost contact structures. There is an alternative perspective on generalization of contact structures, stemming from Sasaki's study on complex structures. When $M$ is a $2 n$-dimensional manifold and $J$ is a real linear bundle map from the tangent bundle $T M$ to itself such that $J \circ J=-\mathrm{Id}$, then $J$ is called an almost complex structure. Recall that the Nijenhuis tensor $N_{J}$ of an almost complex structure is

$$
N_{J}\left(X_{1}, X_{2}\right)=\left[J X_{1}, J X_{2}\right]+J^{2}\left[X_{1}, X_{2}\right]-J\left(\left[J X_{1}, X_{2}\right]+\left[X_{1}, J X_{2}\right]\right)
$$

for all vector fields $X_{1}$ and $X_{2}$. If $N_{J}$ is equal to zero identically, then $J$ is called a complex structure. It is well known that the vanishing of the Nijenhuis tensor is equivalent to the manifold $M$ being a complex manifold. For any almost complex structure, through an averaging process one could easily construct an almost Hermitian structure, i.e. an almost complex structure with a Riemannian metric $g$ such that $g\left(J X_{1}, J X_{2}\right)=g\left(X_{1}, X_{2}\right)$ for any tangent vectors $X_{1}$ and $X_{2}$.

In developing a generalization of complex structures for odd-dimensional manifolds, Sasaki discovered what is known as almost contact structure today [1] [19]. Suppose that $M$ is a $(2 \mathrm{n}+1)$-dimensional manifold with a vector field $F$, a 1 -form $\eta$ and a type (1,1)-tensor $\varphi$ satisfying

$$
\varphi^{2}=-\mathrm{Id}+\eta \otimes F \quad \text { and } \quad \eta(F)=1 .
$$

In his first two publications on this subject in 1961, Sasaki called this triple of tensors simply a $(\varphi, F, \eta)$-structure [19] [21]. With a remark due to Hatakeyama [19, Page 460], Sasaki provided a proof that the existence of such a structure is equivalent to the reducibility of the principal bundle of frames from $G L(2 n+1, \mathbb{R})$ to $\{1\} \times U(n)$. To be precise, given the triple $(\varphi, F, \eta)$, there exists a Riemannian metric $g$ such that

$$
g(\varphi X, \varphi Y)=g(X, Y)-\eta(X) \eta(Y)
$$


Such a metric is called "compatible" with the given structure, and it is not unique [1] [19]. In addition, any quadruple $(\varphi, F, \eta, g)$ satisfying (2) and (3) is equivalent to a reduction of the principal bundle of frames to a $\{1\} \times U(n)$-bundle.

On the other hand, it is known for a long time that the structure group of a $(2 n+1)$-dimensional contact manifold "can" be reduced to $\{1\} \times U(n)[3]$. In fact, J. Gray defined an almost contact manifold as a manifold whose principal bundle of frames "can" be reduced from $G L(2 n+1, \mathbb{R})$ to $\{1\} \times U(n)[10]$. To see explicitly that a contact structure induces a $(\varphi, F, \eta)$-structure in Sasaki's sense, one simply finds a Riemannian metric $g$ on the manifold $M$ and an (1,1)-tensor $\varphi$ on $M$ such that

$$
\eta(X)=g(X, F), \quad d \eta(X, Y)=g(X, \varphi(Y))
$$

Considering Sasaki and Hatakeyama's observation above as a tensorial realization of Gray's definition of almost contact structures, many address Sasaki's $(\varphi, F, \eta)$-structure as an almost contact structure [1]. After all, Sasaki himself addressed his $(\varphi, F, \eta)$-structure as an almost contact structure as soon as 1962 [20] [22]. We shall follow Sasaki's convention, but caution our readers once again that a contact structure does not yield Sasaki's triple of tensors until we choose a compatible metric as noted in (4). Likewise, an almost contact structure as given in terms of Sasaki's triple of tensors is not a $\{1\} \times U(n)$-structure until we choose a compatible metric as defined in (3).

When one does choose a compatible metric $g$, one may extend the quadruple of tensors $(\varphi, F, \eta, g)$ on the odd-dimensional manifold $M$ to obtain an almost Hermitian structure on the cone over $M$ Sasaki further analyzed the integrability of this almost complex structure on the cone [21]. The integrability over the cone yields the concept of "normal contact structures" or "normal almost contact structures" [1] [21]. If one defines

$$
N_{\varphi}\left(X_{1}, X_{2}\right)=\left[\varphi X_{1}, \varphi X_{2}\right]+\varphi^{2}\left[X_{1}, X_{2}\right]-\varphi\left(\left[\varphi X_{1}, X_{2}\right]+\left[X_{1}, \varphi X_{2}\right]\right)
$$

for all vector fields $X_{1}$ and $X_{2}$, then an almost contact structure $(\varphi, F, \eta)$ is normal if and only if

$$
N_{\varphi}=-F \otimes d \eta, \quad L_{F} \varphi=0 \quad \text { and } \quad L_{F} \eta=0 .
$$

With a choice of compatible metric, this line of development yields the theory of Sasakian geometry, which is considered an odd-dimensional analogue of Kählerian 
geometry. This subject enjoys significant progress in the past ten years. See the book by Boyer and Galicki [2], and references therein.

2.3. Remarks. The above review demonstrates that to realize a contact structure as an almost contact structure or Sasaki's $(\varphi, F, \eta)$-structure, we need to choose a compatible metric. On the other hand, the inclusion of contact forms as a subclass of almost cosymplectic structure is straightforward and unique.

Therefore, we adopt Libermann's perspective: the concept of almost cosymplectic structures naturally generalizes the concept of symplectic structures from even to odd-dimension. It includes contact forms as natural examples. We also adopt Sasaki's early perspective: the concept of almost contact structures naturally generalizes the concept of complex structures from even to odd-dimension.

In classical geometry, the bridge between these two perspectives is a choice of compatible metrics. In the rest of this article, we shall see that the concepts of almost cosymplectic structures and almost contact structures could be unified without explicit metric geometry. It is due to one's ability to handle tangent bundle and cotangent bundle simultaneously in "generalized geometry", as developed by Hitchin, Gualtieri and many others in the past five years.

\section{Some ReCEnt Developments}

In the past few years, a major development in analyzing complex structures and symplectic structures is to put them within a single category of "generalized complex structures" [9] [11]. It is best understood in terms of Courant algebroids.

For a manifold $M$ of any dimension, consider the vector bundle $T M \oplus T^{*} M \rightarrow$ $M$. Its space of local sections is endowed with two natural $\mathbb{R}$-bilinear operations.

- A symmetric bilinear form $\langle\cdot, \cdot\rangle$ is defined by

$$
\langle X+\alpha, Y+\beta\rangle=\alpha(Y)+\beta(X) .
$$

- The Courant bracket is given by

$$
\llbracket X+\alpha, Y+\beta \rrbracket=[X, Y]+L_{X} \beta-L_{Y} \alpha-\frac{1}{2} d\left(\iota_{X} \beta-\iota_{Y} \alpha\right) .
$$


3.1. Generalized complex structures. Suppose that $M$ is even-dimensional. A generalized almost complex structure on $M$ is a bundle automorphism $\mathcal{I}$ : $T M \oplus T^{*} M \rightarrow T M \oplus T^{*} M$ such that $\mathcal{I}^{2}=-\mathrm{Id}$ and $\mathcal{I}^{*}+\mathcal{I}=0$. Due to the last condition, a generalized almost complex structure $\mathcal{I}$ on $M$ can be written in a matrix form respecting the direct sum decomposition:

$$
\mathcal{I}=\left(\begin{array}{ll}
\varphi & \pi^{\sharp} \\
\theta^{b}-\varphi^{*}
\end{array}\right),
$$

where $\varphi$ is a (1,1)-tensor field, $\pi$ a bivector field on $M, \theta$ a 2 -form on $M$. Here we adopt the following convention.

$$
\left(\pi^{\sharp} \alpha\right)(\beta)=\pi(\alpha, \beta), \quad \text { and } \quad Y\left(\theta^{b} X\right)=\theta(X, Y)
$$

for any 1-forms $\alpha$ and $\beta$, 2-form $\theta$, bivector field $\pi$, and vector fields $X$ and $Y$.

The condition $\mathcal{I} \circ \mathcal{I}=-\mathrm{Id}$ could be expressed in terms of the components of $\mathcal{I}$.

$$
\varphi^{2}+\pi^{\sharp} \theta^{b}=-\mathrm{Id}, \quad \varphi \pi^{\sharp}=\pi^{\sharp} \varphi^{*}, \quad \theta^{b} \varphi=\varphi^{*} \theta^{b} .
$$

The same condition implies that the complexification of the bundle $T M \oplus T^{*} M$ splits into a direct sum of $( \pm i)$-eigenbundles. The condition $\mathcal{I}^{*}+\mathcal{I}=0$ implies that these bundles are maximal isotropic with respect to the bilinear form (7).

Definition 3.1. [9] A generalized almost complex structure $\mathcal{I}$ is integrable if it satisfies the condition $N_{\mathcal{I}}=0$, where

$$
N_{\mathcal{I}}\left(e_{1}, e_{2}\right):=\llbracket \mathcal{I} e_{1}, \mathcal{I} e_{2} \rrbracket+\mathcal{I}^{2} \llbracket e_{1}, e_{2} \rrbracket-\mathcal{I}\left(\llbracket \mathcal{I} e_{1}, e_{2} \rrbracket+\llbracket e_{1}, \mathcal{I} e_{2} \rrbracket\right)
$$

for all sections $e_{1}, e_{2}$ of the vector bundle $T M \oplus T^{*} M$ over the manifold $M$.

The integrability of $\mathcal{I}$ could be expressed in terms of its components. Consider the bracket $\{,\}_{\pi}$ on 1 -forms defined by

$$
\{\alpha, \beta\}_{\pi}=L_{\pi^{\sharp} \alpha} \beta-L_{\pi^{\sharp} \beta} \alpha-d(\pi(\alpha, \beta)) .
$$

Proposition 3.2. [5] Let $I=(\varphi, \pi, \theta)$ be a generalized almost complex structure on $M$. Then $I$ is integrable if and only if the following conditions are satisfied:

(C1) $\pi$ is a Poisson bivector field, i.e. $[\pi, \pi]=0$.

(C2) $\varphi^{*}\left(\{\alpha, \beta\}_{\pi}\right)=L_{\pi^{\sharp} \alpha}\left(\varphi^{*} \beta\right)-L_{\pi^{\sharp} \beta}\left(\varphi^{*} \alpha\right)-d\left(\pi\left(\varphi^{*} \alpha, \beta\right)\right)$; for all 1-forms $\alpha$ and $\beta$.

(C3) $\mathcal{N}_{\varphi}(X, Y)=\pi^{\sharp}\left(\iota_{X \wedge Y} d \theta\right)$, for all vector fields $X$ and $Y$; 
(C4) $d \theta_{\varphi}(X, Y, Z)=d \theta(\varphi X, Y, Z)+d \theta(X, \varphi Y, Z)+d \theta(X, Y, \varphi Z)$, for all vector fields $X, Y$ and $Z$, when $\theta_{\varphi}(X, Y)=\theta(\varphi X, Y)$.

3.2. Generalized almost contact structures. In view of the definition of almost contact structures, we modify the definition of the analogues of generalized complex structures for odd-dimensional manifolds. For another equivalent definition, see [23].

Definition 3.3. A generalized almost contact structure consists of a bundle endomorphism $\Phi$ from $T M \oplus T^{*} M$ to itself and a section $F+\eta$ of $T M \oplus T^{*} M$ such that

(1) $\Phi+\Phi^{*}=0$,

(2) $\eta(F)=1, \Phi(F)=0$ and $\Phi(\eta)=0$,

(3) $\Phi \circ \Phi=-\mathrm{Id}+F \odot \eta$,

where $F \odot \eta$ is the bundle map from $T M \oplus T^{*} M$ to itself given by

$$
F \odot \eta(X+\alpha):=F \eta(X)+\eta \alpha(F)
$$

In terms of the direct sum decomposition of $T M \oplus T^{*} M$, the first condition in the definition is equivalent to the existence of a $(1,1)$-tensor $\varphi$, a 2 -form $\theta$ and a bivector field $\pi$ such that

$$
\Phi=\left(\begin{array}{cc}
\varphi & \pi^{\sharp} \\
\theta^{b} & -\varphi^{*}
\end{array}\right),
$$

where we use the convention of (10). The other conditions could be expressed in terms of the components of $\Phi$, the vector field $F$ and the 1-form $\eta$.

$$
\begin{gathered}
\theta^{b} \varphi=\varphi^{*} \theta^{b}, \quad \varphi \pi^{\sharp}=\pi^{\sharp} \varphi^{*}, \\
\varphi^{2}+\pi^{\sharp} \theta^{b}=-\mathrm{Id}+F \otimes \eta, \quad \text { and } \quad\left(\varphi^{*}\right)^{2}+\theta^{b} \pi^{\sharp}=-\mathrm{Id}+\eta \otimes F . \\
\eta \circ \varphi=\varphi^{*} \eta=0, \quad \eta \circ \pi^{\sharp}=\pi^{\sharp} \eta=0, \quad \iota_{F} \varphi=0, \quad \iota_{F} \theta=0, \quad \iota_{F} \eta=1 .
\end{gathered}
$$

In subsequent discussion, we often address a generalized almost contact structure in terms of its components and denote it by $J=(F, \eta, \pi, \theta, \varphi)$. 
3.3. Examples. Consider a manifold $M$ with a Sasaki's triple $(\varphi, F, \eta)$ as an almost contact structure. It is apparent that if we set

$$
\Phi=\left(\begin{array}{cc}
\varphi & 0 \\
0 & -\varphi^{*}
\end{array}\right),
$$

we have a generalized almost contact structure, with the 2-form $\theta$ and bivector field $\pi$ being equal to zero.

Given a manifold $M$ with as an almost cosymplectic structure $(\eta, \theta)$, we could construct the components of a generalized almost contact structure in terms of $\eta$ and $\theta$ as follows.

Let $F$ be the unique vector field such that $\iota_{F} \theta=0$ and $\iota_{F} \eta=1$. Again, we call this vector field the Reeb field. Consider the map $b: T M \rightarrow T^{*} M$ defined by

$$
b(X):=\iota_{X} \theta-\eta(X) \eta .
$$

Since $\eta \wedge \theta^{n} \neq 0$ everywhere, $b$ is an isomorphism. Define a bivector field $\pi$ by its contraction with two 1 -forms. i.e.

$$
\pi(\alpha, \beta):=\theta\left(b^{-1}(\alpha), b^{-1}(\beta)\right) .
$$

In particular, the vector $\pi^{\sharp}(\alpha)$ is defined by

$$
\pi^{\sharp}(\alpha)(\beta)=\pi(\alpha, \beta)=\theta\left(b^{-1}(\alpha), b^{-1}(\beta)\right) .
$$

It is now an elementary computation to verify the next lemma.

Lemma 3.4. When $b(X)=\alpha, \pi^{\sharp}(\alpha)=-X+\eta(X) F$. In particular, $\pi^{\sharp}(\eta)=0$ and $\pi^{\sharp}\left(\iota_{X} \theta\right)=-X+\eta(X) F$. Moreover, $b$ is an isomorphism from ker $F$ to ker $\eta$, and $-\pi^{\sharp}$ is its inverse.

Given the above information, it is now straightforward to see that the tensors $J=(F, \eta, \pi, \theta, \varphi)$, with $\varphi=0$, define a generalized almost contact structure. The associated bundle map $\Phi$ is given by

$$
\Phi=\left(\begin{array}{cc}
0 & \pi^{\sharp} \\
\theta^{b} & 0
\end{array}\right) .
$$

Since every contact form $\eta$ determines an almost cosymplectic structure $(\eta, d \eta)$, it uniquely determines a generalized almost contact structure. We will study contact forms from such perspective. 
3.4. Alternative approach. Before we investigate the integrability of generalized almost contact structures, we briefly review its relation with a different definition due to the second author and her collaborator [12].

On an odd-dimensional smooth manifold $M$, set $E^{1}(M)=(T M \times \mathbb{R}) \oplus\left(T^{*} M \times\right.$ $\mathbb{R})$. One may consider a bundle map $\widetilde{I}: E^{1}(M) \rightarrow E^{1}(M)$ such that $\widetilde{I} \circ \widetilde{I}=-\mathrm{Id}$ and $\widetilde{I}+\widetilde{I}^{*}=0$. Such object is used to be called generalized almost contact structure [12]. This definition is equivalent to the one given in [12, Definition 4.1], in terms of maximal isotropic subbundles of $E^{1}(M)$ with respect to a natural symmetric pairing. With respect to the direct sum decomposition of $E^{1}(M)$, the map $\widetilde{I}$ decomposes as follows:

$$
\widetilde{I}=\left(\begin{array}{cc}
N & P^{\sharp} \\
\Theta^{b} & -N^{*}
\end{array}\right)
$$

where the four components of $\widetilde{I}$ are:

- a bundle map $N: T M \times \mathbb{R} \rightarrow T M \times \mathbb{R}$;

- a skew-symmetric bundle map $P^{\sharp}: T^{*} M \times \mathbb{R} \rightarrow T M \times \mathbb{R}$ given by a bivector field $\pi$ and a vector field $F$ such that for any 1-form $\alpha$ and function $g$ on the manifold $M, P^{\sharp}(\alpha, g)=\left(\pi^{\sharp} \alpha-g F, \iota_{F} \alpha\right)$;

- a skew-symmetric bundle map $\Theta^{b}: T M \times \mathbb{R} \rightarrow T^{*} M \times \mathbb{R}$ given by a 2-form $\theta$ and a 1-form $\eta$ such that for any vector field $X$ and function $f$ on the manifold $M, \Theta^{b}(X, f)=\left(\iota_{X} \theta-f \eta, \iota_{X} \eta\right)$.

When $J=(F, \eta, \pi, \theta, \varphi)$ is a generalized almost contact structure as given in Definition 3.3, we set

$$
N=\left(\begin{array}{cc}
\varphi & 0 \\
0 & 0
\end{array}\right), \quad P^{\sharp}=\left(\begin{array}{cc}
\pi^{\sharp}-F \\
F & 0
\end{array}\right), \quad \Theta^{b}=\left(\begin{array}{cc}
\theta^{b} & -\eta \\
\eta & 0
\end{array}\right) .
$$

This construction makes every generalized almost contact structure as given in Definition 3.3 a generalized almost contact structure in the sense of Wade et. al. [12]. From now on, we adopt Definition 3.3 as our definition of generalized almost contact structures. 


\section{Integrability, A SASAKIAN APPROACH}

Next we explore possible definitions for integrability of generalized almost contact structures. In this section, we examine an approach similar to the development of "normal contact structures" as found in [21] [22] (see also [1]).

4.1. Integrability over a cone. Let $M$ be a smooth odd-dimensional manifold. Consider the cone $C(M)=M \times \mathbb{R}^{+}$, where $\mathbb{R}^{+}$is the positive half-line with its unit parameter $t$. Given a generalized almost contact structure $J=(F, \eta, \pi, \theta, \varphi)$, define the following tensors on $C(M)$ :

$$
\widetilde{P}=\frac{1}{t^{2}} \pi+\frac{1}{2 t} F \wedge \frac{\partial}{\partial t}, \quad \widetilde{\Theta}=t^{2} \theta+2 t \eta \wedge d t .
$$

We extend $\varphi$ by setting

$$
\widetilde{N}_{\mid T M}=\varphi, \quad \widetilde{N}\left(\frac{\partial}{\partial t}\right)=0 .
$$

Consider the map $\widetilde{J}: T C(M) \oplus T^{*} C(M) \rightarrow T C(M) \oplus T^{*} C(M)$ defined by:

$$
\widetilde{J}=\left(\begin{array}{cc}
\widetilde{N} & \widetilde{P} \\
\widetilde{\Theta} & -\widetilde{N}^{*}
\end{array}\right) .
$$

It becomes a generalized almost complex structure on the cone $C(M)$.

Definition 4.1. A generalized almost contact structure $J$ on $M$ is said to be integrable on the cone over $M$ if the corresponding generalized almost complex structure $\widetilde{J}$ on the cone $C(M)$ is integrable as a generalized almost complex structure.

Theorem 4.2. A generalized almost contact structure $J=(F, \eta, \pi, \theta, \varphi)$ on a manifold $M$ is integrable on the cone over $M$ if and only if its components satisfy the following conditions.

(A1) $[\pi, \pi]=2 F \wedge \pi$, and $[F, \pi]=0$.

(A2) $L_{F} \varphi=0, L_{F} \theta=0$, and $L_{F} \eta=0$.

(A3) For any 1-forms $\alpha$ and $\beta, \varphi^{*}\{\alpha, \beta\}_{\pi}=L_{\pi^{\sharp} \alpha}\left(\varphi^{*} \beta\right)-L_{\pi^{\sharp} \beta}\left(\varphi^{*} \alpha\right)-d \pi\left(\varphi^{*} \alpha, \beta\right)$.

(A4) For any vector fields $X$ and $Y, \pi^{\sharp}\left(\iota_{X} d \eta-\iota_{X} \theta\right)=0$ and

$$
N_{\varphi}(X, Y)=\pi^{\sharp}\left(\iota_{X \wedge Y} d \theta\right)+(\theta(X, Y)-d \eta(X, Y)) F .
$$

(A5) For any vector fields $X, Y, Z$, define $\theta_{\varphi}(X, Y):=\theta(\varphi X, Y)$. Then

$$
d \theta_{\varphi}(X, Y, Z)=d \theta(\varphi X, Y, Z)+d \theta(X, \varphi Y, Z)+d \theta(X, Y, \varphi Z) .
$$


Proof: Property $(\mathrm{C} 1)$ of Proposition 3.2 says that $[\widetilde{P}, \widetilde{P}]=0$. But, $[\widetilde{P}, \widetilde{P}]=0$ if and only if $[\pi, \pi]-2 F \wedge \pi=0$ and $[F, \pi]=0$. From Property (C2), one gets $\widetilde{N}^{*}\left(\{2 t d t, \beta\}_{\widetilde{P}}\right)=L_{F}\left(\varphi^{*} \beta\right)$. Since $\varphi^{*}\left(L_{F} \beta\right)=L_{F}\left(\varphi^{*} \beta\right)$ for any 1 -form $\beta$, integrability implies that $L_{F} \varphi=0$. Property (C3) ensures that for any vector fields $X$ and $Y$,

$$
0=\left\langle N_{\widetilde{N}}(X, Y), 2 t d t\right\rangle=t^{2}\left\langle F, \iota_{X \wedge Y} d \theta\right\rangle .
$$

Since $\iota_{F} \theta=0$, it follows that $L_{F} \theta=0$. Equation (A3) is obtained from a direct computation using $(\mathrm{C} 2)$. In addition,

$$
0=N_{\widetilde{N}}\left(X, \frac{1}{2 t} \frac{\partial}{\partial t}\right)=t^{2} \pi^{\sharp}\left(\iota_{X} d \eta-\iota_{X} \theta\right)+\frac{1}{2 t} d \eta(X, F) \frac{\partial}{\partial t},
$$

for all vector field $X$ on $M$. Therefore,

$$
\iota_{F} d \eta=0 \quad \text { and } \quad \pi^{\sharp}\left(\iota_{X} d \eta-\iota_{X} \theta\right)=0 .
$$

The second part of (A4) is obtained by applying (C3) to vector fields $X$ and $Y$ on $M$. Finally, the last relation comes from (C4) when applied to $\widetilde{N}$ and $\widetilde{\theta}$.

The integrability condition in Theorem 4.2 is, in some sense, more general than the one given by Vaisman in [23]. For instance, in our case the bivector field $\pi$ and the vector field $F$ together form a Jacobi structure, and hence $\pi$ is a not necessarily a Poisson structure.

4.2. Examples. Recall that an almost cosymplectic structure $(\theta, \eta)$ could be realized as a generalized almost contact structure with $\varphi=0$. Therefore, the integrability conditions are reduced.

(1) $[\pi, \pi]=2 F \wedge \pi$ and $[F, \pi]=0$.

(2) $L_{F} \theta=\iota_{F} d \theta=0$.

(3) $\theta, \pi, \eta$ and $F$ satisfy the relations: for all vector fields $X$ and $Y$

$$
\iota_{F} d \eta=0, \quad \pi^{\sharp}\left(\iota_{X} d \eta-\iota_{X} \theta\right)=0, \quad \pi^{\sharp}\left(\iota_{X \wedge Y} d \theta\right)+(\theta(X, Y)-d \eta(X, Y)) F=0 .
$$

Due to the identity $\pi^{\sharp}\left(\iota_{X} d \eta-\iota_{X} \theta\right)=0$ and Lemma 3.4, for any $X$ there is a function $f$ such that $\iota_{X} d \eta-\iota_{X} \theta=f \eta$. Contracting the last identity with $F$, we have $-\iota_{X} \iota_{F} d \eta=f$. As the integrability condition requires $\iota_{F} d \eta=0$, $f \equiv 0$. Therefore $\iota_{X}(d \eta-\theta)=0$ for all $X$. It follows that $\theta=d \eta$ and all other integrability conditions are then fulfilled. Therefore, we have the following. 
Proposition 4.3. The generalized almost contact structure determined by an almost cosymplectic structure is integrable on the cone if and only if it is a contact structure in the classical sense.

An almost contact structure $(\varphi, \eta, F)$ defines a generalized almost cosymplectic structure with $\pi=0$ and $\theta=0$. The integrability conditions are reduced to

$$
L_{F} \varphi=0, \quad L_{F} \eta=0, \quad N_{\varphi}=-F \otimes d \eta .
$$

Therefore, the following is immediate.

Proposition 4.4. The generalized almost contact structure determined by a classical almost contact structure $(\varphi, F, \eta)$ is integrable on the cone if and only if it is a normal contact structure in the classical sense.

\section{Integrability, a Courant Algebroid Perspective}

With the symmetric pairing in (7), Courant bracket in (8) and the projection onto the first summand as anchor map, $\left(T M \oplus T^{*} M,\langle\cdot, \cdot\rangle, \llbracket \cdot, \cdot \rrbracket\right)$ is a Courant algebroid. Given a generalized almost contact structure $J=(F, \eta, \pi, \theta, \varphi)$, consider the bundle map $\Phi$ on $T M \oplus T^{*} M$ as defined in (14). Denote by $L_{F}$ (resp. $L_{\eta}$ ) the trivial complex line bundle generated by $F$ (resp. $\eta$ ). Let ker $\eta$ be the subbundle of $T M$ such that each fiber is the kernel of $\eta$. Let ker $F$ be the subbundle of $T^{*} M$ such that each fiber consists of 1-forms whose evaluation on $F$ is equal to zero. By Definition 3.3, $\Phi^{2}=-\mathrm{Id}$ on $\operatorname{ker} \eta \oplus \operatorname{ker} F$. Therefore, the complexification of ker $\eta \oplus \operatorname{ker} F$ is the direct sum of the $\pm i$-eigenbundles. Define

$$
E^{(1,0)}=\{e-i \Phi(e) \mid e \in \operatorname{Ker} \eta \oplus \operatorname{Ker} F\}, E^{(0,1)}=\{e+i \Phi(e) \mid e \in \operatorname{Ker} \eta \oplus \operatorname{Ker} F\} .
$$

We have the natural splitting: $\left(T M \oplus T^{*} M\right)_{\mathbb{C}}=L_{F} \oplus L_{\eta} \oplus E^{(1,0)} \oplus E^{(0,1)}$.

In subsequent analysis, the following four different complex vector bundles will play different roles. Namely,

$$
L:=L_{F} \oplus E^{(1,0)}, \quad \bar{L}:=L_{F} \oplus E^{(0,1)}, \quad L^{*}:=L_{\eta} \oplus E^{(0,1)}, \quad \bar{L}^{*}:=L_{\eta} \oplus E^{(1,0)} .
$$

Note that $L_{F}$ is the complexification of a real line bundle, its conjugation is itself. Therefore, the complex conjugation map sends $L$ to $\bar{L}$. 
Definition 5.1. A generalized almost contact structure $J=(F, \eta, \pi, \theta, \varphi)$ is Courant-integrable if the space of sections of bundle $L$ is closed with respect to the Courant bracket.

The condition $\llbracket L, L \rrbracket \subseteq L$ could be expressed in terms of the two components of $L$. Since $L_{F}$ is a rank-1 bundle, it is apparent that $\llbracket L_{F}, L_{F} \rrbracket \subseteq L_{F}$. Therefore, the non-trivial conditions are due to the following two inclusions.

$$
\llbracket L_{F}, E^{(1,0)} \rrbracket \subseteq L_{F} \oplus E^{(1,0)}, \quad \llbracket E^{(1,0)}, E^{(1,0)} \rrbracket \subseteq L_{F} \oplus E^{(1,0)} .
$$

Note that although the closure of the space of sections of $L$ with respect to the Courant bracket is equivalent to the closure of $\bar{L}$ by conjugation, a lack of natural isomorphism between $\bar{L}$ and $L^{*}$ means that the space of sections of $L^{*}$ is not necessarily closed when $L$ is.

5.1. Integrability of almost cosymplectic structures. As noted in Section 3.3 , given an almost cosymplectic structure $(\eta, \theta)$, the corresponding generalized almost contact structure has $\varphi=0$. When $X+\alpha$ is a section of $\operatorname{ker} \eta \oplus \operatorname{ker} F$, $\Phi(X+\alpha)=\pi^{\sharp}(\alpha)+\theta^{b}(X)$.

To examine when the bundle $L_{F} \oplus E^{(1,0)}$ is closed with respect to the Courant bracket, we first consider $\llbracket L_{F}, E^{(1,0)} \rrbracket$. When $X$ is a section of ker $\eta$,

$$
\llbracket F, X-i \Phi(X) \rrbracket=\llbracket F, X-i \theta^{b}(X) \rrbracket=\left(L_{F} X\right)-i \theta^{b}\left(L_{F} X\right)+\iota_{X}\left(L_{F} \theta\right) .
$$

Similarly, if $\alpha$ is a section of $\operatorname{ker} F$, let $X=b(\alpha)$. By Lemma 3.4

$$
\begin{aligned}
& \llbracket F, \alpha-i \Phi(\alpha) \rrbracket=\llbracket F, \alpha-i \pi^{\sharp}(\alpha) \rrbracket=i \llbracket F, X-i \theta^{b}(X) \rrbracket \\
= & i\left(\left(L_{F} X\right)-i \theta^{b}\left(L_{F} X\right)+\iota_{X}\left(L_{F} \theta\right)\right) .
\end{aligned}
$$

Therefore, the analysis of $\llbracket F, \alpha-i \Phi(\alpha) \rrbracket$ is identical to the one of $\llbracket F, X-i \Phi(X) \rrbracket$.

Since $\theta(F)=0,\left\langle\left(L_{F} X\right)-i \theta^{\mathrm{b}}\left(L_{F} X\right)+\iota_{X}\left(L_{F} \theta\right), F\right\rangle=0$. Since $\eta(X)=0$ and $\eta(F)=1$,

$$
\left\langle\left(L_{F} X\right)-i \theta^{b}\left(L_{F} X\right)+\iota_{X}\left(L_{F} \theta\right), \eta\right\rangle=\eta\left(L_{F} X\right) .
$$

It follows that

$$
\left(L_{F} X\right)-i \theta^{b}\left(L_{F} X\right)+\iota_{X}\left(L_{F} \theta\right)-\eta\left(L_{F} X\right) F
$$


is a section of $\operatorname{ker} \eta \oplus \operatorname{ker} F$. Therefore, $\llbracket L_{F}, E^{(1,0)} \rrbracket \subseteq L$ if and only if the above tensor is a section of $E^{(1,0)}$. Since $\iota_{F} \theta=0$, the above tensor is equal to

$$
\begin{aligned}
& \left(L_{F} X\right)-\eta\left(L_{F} X\right) F-i \theta^{b}\left(L_{F} X-\eta\left(L_{F} X\right) F\right)+\iota_{X}\left(L_{F} \theta\right) \\
= & \left(L_{F} X\right)-\eta\left(L_{F} X\right) F-i \Phi\left(L_{F} X-\eta\left(L_{F} X\right) F\right)+\iota_{X}\left(L_{F} \theta\right) .
\end{aligned}
$$

It is a section of $E^{(1,0)}$ if and only if $\iota_{X}\left(L_{F} \theta\right)$ is a section of $E^{(1,0)}$. As $\iota_{X}\left(L_{F} \theta\right)$ is a real section, it is possible only when $\iota_{X}\left(L_{F} \theta\right)=0$ for all $X$ in $\operatorname{ker} \eta$. Since $\theta(F)=0$, we always have $\iota_{F}\left(L_{F} \theta\right)=0$. Therefore,

$$
\llbracket L_{F}, E^{(1,0)} \rrbracket \subseteq L \quad \text { if and only if } \quad L_{F} \theta=0 .
$$

Next we identify the conditions for $\llbracket E^{(1,0)}, E^{(1,0)} \rrbracket \subseteq L$. A priori, there are three cases to consider. If $X$ and $Y$ are sections of $\operatorname{ker} \eta$, then

$$
\begin{aligned}
& \llbracket X-i \Phi(X), Y-i \Phi(Y) \rrbracket=\llbracket X-i \theta^{b}(X), Y-i \theta^{b}(Y) \rrbracket \\
= & \llbracket X, Y \rrbracket-i\left(\llbracket X, \theta^{b}(Y) \rrbracket-\llbracket Y, \theta^{b}(X) \rrbracket\right) \\
= & \llbracket X, Y \rrbracket-i \theta^{b}(\llbracket X, Y \rrbracket)+i \theta^{b}(\llbracket X, Y \rrbracket) \\
& -i\left(\iota_{X} d \theta^{b}(Y)+d \iota_{X} \theta^{b}(Y)-\frac{1}{2} d \iota_{X} \theta^{b}(Y)-\iota_{Y} d \theta^{b}(X)-d \iota_{Y} \theta^{b}(X)+\frac{1}{2} d \iota_{Y} \theta^{b}(X)\right) \\
= & \llbracket X, Y \rrbracket-i \Phi(\llbracket X, Y \rrbracket)-i\left(-\theta^{b}(\llbracket X, Y \rrbracket)+\iota_{X} d \theta^{b}(Y)-\iota_{Y} d \theta^{b}(X)-d(\theta(X, Y))\right) \\
= & \llbracket X, Y \rrbracket-i \Phi(\llbracket X, Y \rrbracket)-i \iota_{Y} \iota_{X} d \theta .
\end{aligned}
$$

Suppose that $\alpha$ and $\beta$ are sections of ker $\eta$. Let $X$ and $Y$ be vector fields such that $\theta^{b}(X)=b(X)=\alpha$ and $\theta^{b}(Y)=b(Y)=\beta$. By Lemma 3.4, $X$ and $Y$ are in ker $\eta, \pi^{\sharp}(\alpha)=-X$ and $\pi^{\sharp}(\beta)=-Y$. It follows that

$$
\begin{aligned}
& \llbracket \alpha-i \Phi(\alpha), \beta-i \Phi(\beta) \rrbracket=\llbracket \alpha-i \pi^{\sharp}(\alpha), \beta-i \pi^{\sharp}(\beta) \rrbracket=\llbracket \alpha+i X, \beta+i Y \rrbracket \\
= & \llbracket \theta^{b}(X)+i X, \theta^{b}(Y)+i Y \rrbracket=-(\llbracket X, Y \rrbracket-i \Phi(\llbracket X, Y \rrbracket))+i \iota_{Y} \iota_{X} d \theta .
\end{aligned}
$$

Finally, suppose that $X$ is a section of $\operatorname{ker} \eta$ and $\beta$ is a section of $\operatorname{ker} F$. Then

$$
\begin{aligned}
& \llbracket X-i \Phi(X), \beta-i \Phi(\beta) \rrbracket \\
= & \llbracket X-i \theta^{b}(X), \beta-i \pi^{\sharp}(\beta) \rrbracket=\llbracket X-i \theta^{b}(X), \theta^{b}(Y)+i Y \rrbracket \\
= & i \llbracket X-i \theta^{b}(X), Y-i \theta^{b}(Y) \rrbracket=i(\llbracket X, Y \rrbracket-i \Phi(\llbracket X, Y \rrbracket))+\iota_{Y} \iota_{X} d \theta .
\end{aligned}
$$

Therefore, $\llbracket E^{(1,0)}, E^{(1,0)} \rrbracket \subseteq L$ if and only if for all sections $X$ and $Y$ of ker $\eta$,

$$
\llbracket X, Y \rrbracket-i \theta^{b}(\llbracket X, Y \rrbracket)-i \iota_{Y} \iota_{X} d \theta
$$


is a section of $L$. Since $\theta(F)=0$,

$$
\left\langle\llbracket X, Y \rrbracket-i \Phi(\llbracket X, Y \rrbracket)-i \iota_{Y} \iota_{X} d \theta, F\right\rangle=-i\left(\iota_{F} d \theta\right)(X, Y)=-i \iota_{Y} \iota_{X}\left(L_{F} \theta\right) .
$$

It is equal to zero due to the constraint in (28). Therefore, removing the $F$ component from (29), we get a section of $\operatorname{ker} \eta \oplus \operatorname{ker} F$. It is

$$
\begin{aligned}
& \llbracket X, Y \rrbracket-i \theta^{b}(\llbracket X, Y \rrbracket)-i \iota_{Y} \iota_{X} d \theta-\left\langle\llbracket X, Y \rrbracket-i \theta^{b}(\llbracket X, Y \rrbracket)-i \iota_{Y} \iota_{X} d \theta, \eta\right\rangle F \\
= & \llbracket X, Y \rrbracket-i \theta^{b}(\llbracket X, Y \rrbracket)-i \iota_{Y} \iota_{X} d \theta-\eta(\llbracket X, Y \rrbracket) F \\
= & \llbracket X, Y \rrbracket-\eta(\llbracket X, Y \rrbracket) F-i \theta^{b}(\llbracket X, Y \rrbracket-\eta(\llbracket X, Y \rrbracket) F)-i \iota_{Y} \iota_{X} d \theta
\end{aligned}
$$

It is a section of $L$ if and only if $\iota_{Y} \iota_{X} d \theta$ is a section of $E^{(1,0)}$. Since we could choose $X$ and $Y$ to be any real sections of ker $\eta, \iota_{Y} \iota_{X} d \theta$ could be a section of $E^{(1,0)}$ only when it is zero. As $\iota_{F} d \theta=0$. The section in $(30)$ is in $E^{(1,0)}$ for any $X$ and $Y$ in $\operatorname{ker} \eta$ if and only if $d \theta=0$ everywhere.

Proposition 5.2. The generalized almost contact structure defined by a classical almost cosymplectic structure $(\eta, \theta)$ is Courant-integrable if and only if $d \theta=0$. In particular, any contact structure $(\eta, d \eta)$ as an almost cosymplectic structure is Courant-integrable.

\subsection{Integrability of almost contact structures.}

Proposition 5.3. The generalized almost contact structure associated to an almost contact structure $(\varphi, F, \eta)$ is Courant-integrable if and only if $\left(L_{F} \varphi\right) X$ is a section of $L_{F}$ and $N_{\varphi}(X, Y)=-d \eta(X, Y) F$ for any vector fields $X$ and $Y$ of ker $\eta$.

Proof: As a generalized almost contact structure, a classical almost contact structure is given by $\theta=0$ and $\pi=0$. The map $\Phi$ in this case is given by (18).

To consider $\llbracket L_{F}, E^{(1,0)} \rrbracket$, let $X$ be a section of ker $\eta$. Then

$$
\llbracket F, X-i \Phi(X) \rrbracket=L_{F} X-i L_{F}(\varphi X)=L_{F} X-i \varphi\left(L_{F} X\right)-i\left(L_{F} \varphi\right)(X) .
$$

It is a vector field. Its $L_{F}$-component is

$$
\eta\left(L_{F} X-i L_{F}(\varphi X)\right) F \text {. }
$$

After it is removed, we have a section in $\operatorname{ker} \eta \oplus \operatorname{ker} F$ :

$$
L_{F} X-\eta\left(L_{F} X\right) F-i L_{F}(\varphi X)+i \eta\left(L_{F}(\varphi X)\right) F .
$$


It is a section of $E^{(1,0)}$ if and only if

$$
L_{F}(\varphi X)-\eta\left(L_{F}(\varphi X)\right) F=\varphi\left(L_{F} X-\eta\left(L_{F} X\right) F\right)=\varphi\left(L_{F} X\right) .
$$

Equivalently, for any section $X$ of $\operatorname{ker} \eta$,

$$
\left(L_{F} \varphi\right) X=\eta\left(L_{F}(\varphi X)\right) F=\eta\left(\left(L_{F} \varphi\right) X\right) F
$$

i.e. $L_{F} \varphi: \operatorname{ker} \eta \rightarrow L_{F}$.

To derive further constraints on integrability, let $\alpha$ be a section of $\operatorname{ker} F$. Making use of the last constraint,

$$
\begin{aligned}
& \llbracket F, \alpha-i \Phi(\alpha) \rrbracket=L_{F} \alpha+i L_{F}\left(\varphi^{*} \alpha\right)=L_{F} \alpha+i \varphi^{*}\left(L_{F} \alpha\right)+i\left(L_{F} \varphi\right)^{*} \alpha \\
= & L_{F} \alpha-i \Phi\left(L_{F} \alpha\right)+i\left(L_{F} \varphi\right)^{*} \alpha=L_{F} \alpha-i \Phi\left(L_{F} \alpha\right) .
\end{aligned}
$$

Since $\left(L_{F} \alpha\right) F=0, L_{F} \alpha-i \Phi\left(L_{F} \alpha\right)$ is a section of $E^{(1,0)}$. Combining the above two cases, we find that

$$
\llbracket L_{F}, E^{(1,0)} \rrbracket \subseteq L \quad \Leftrightarrow \quad\left(L_{F} \varphi\right) X \in L_{F} .
$$

For $\llbracket E^{(1,0)}, E^{(1,0)} \rrbracket$, there are two cases to consider. Suppose that $X$ and $Y$ are sections of $\operatorname{ker} \eta$.

$$
\llbracket X-i \varphi X, Y-i \varphi Y \rrbracket=\llbracket X, Y \rrbracket-\llbracket \varphi X, \varphi Y \rrbracket-i \llbracket X, \varphi Y \rrbracket-i \llbracket \varphi X, Y \rrbracket .
$$

After its $L_{F}$-component is removed, it is equal to

$$
\begin{aligned}
& \llbracket X, Y \rrbracket-\llbracket \varphi X, \varphi Y \rrbracket-i \llbracket X, \varphi Y \rrbracket-i \llbracket \varphi X, Y \rrbracket \\
& -\eta(\llbracket X, Y \rrbracket) F+\eta(\llbracket \varphi X, \varphi Y \rrbracket) F+i \eta(\llbracket X, \varphi Y \rrbracket) F+i \eta(\llbracket \varphi X, Y \rrbracket) F .
\end{aligned}
$$

We require its $E^{(0,1)}$-component vanishes. i.e.

$$
\begin{aligned}
& \llbracket X, Y \rrbracket-\llbracket \varphi X, \varphi Y \rrbracket-i \llbracket X, \varphi Y \rrbracket-i \llbracket \varphi X, Y \rrbracket \\
& -\eta(\llbracket X, Y \rrbracket) F+\eta(\llbracket \varphi X, \varphi Y \rrbracket) F+i \eta(\llbracket X, \varphi Y \rrbracket) F+i \eta(\llbracket \varphi X, Y \rrbracket) F \\
& +i \varphi(\llbracket X, Y \rrbracket-\llbracket \varphi X, \varphi Y \rrbracket-i \llbracket X, \varphi Y \rrbracket-i \llbracket \varphi X, Y \rrbracket \\
& -\eta(\llbracket X, Y \rrbracket) F+\eta(\llbracket \varphi X, \varphi Y \rrbracket) F+i \eta(\llbracket X, \varphi Y \rrbracket) F+i \eta(\llbracket \varphi X, Y \rrbracket) F)=0 .
\end{aligned}
$$

Making use of definition of $N_{\varphi}$ and $\varphi(F)=0$, we find the real part being equal to

$$
\begin{aligned}
& \llbracket X, Y \rrbracket-\llbracket \varphi X, \varphi Y \rrbracket-\eta(\llbracket X, Y \rrbracket) F+\eta(\llbracket \varphi X, \varphi Y \rrbracket) F+\varphi(\llbracket X, \varphi Y \rrbracket+\llbracket \varphi X, Y \rrbracket) \\
= & -N_{\varphi}(X, Y)-\eta(\llbracket X, Y \rrbracket) F .
\end{aligned}
$$


We could similarly compute the imaginary part to find that the $E^{(0,1)}$-part of $\llbracket X-i \varphi X, Y-i \varphi Y \rrbracket$ is equal to

$$
-N_{\varphi}(X, Y)+\eta(\llbracket X, Y \rrbracket) F-i\left(-N_{\varphi}(X, \varphi Y)+\eta(\llbracket X, \varphi Y \rrbracket) F\right) .
$$

As $\varphi$ sends kernel of $\eta$ into kernel of $\eta, \llbracket X-i \varphi X, Y-i \varphi Y \rrbracket$ is contained in $L$ for any sections $X, Y$ of ker $\eta$ if and only if

$$
N_{\varphi}(X, Y)=\eta(\llbracket X, Y \rrbracket) F=-d \eta(X, Y) F .
$$

Next, suppose that $X$ is a section of $\operatorname{ker} \eta$ and $\beta$ is a section of $\operatorname{ker} F$.

$$
\llbracket X-i \varphi X, \beta+i \varphi^{*} \beta \rrbracket=L_{X} \beta+L_{\varphi X}\left(\varphi^{*} \beta\right)-i L_{\varphi X} \beta+i L_{X}\left(\varphi^{*} \beta\right) .
$$

Since it is a differential form, it could be a section of $L$ if and only if it is a section of $E^{(1,0)}$. In particular, it is a section of ker $F$. Given the nature of $X$ and $\beta$,

$\left(L_{X} \beta+L_{\varphi X}\left(\varphi^{*} \beta\right)\right) F=\beta\left(N_{\varphi}(X, F)\right), \quad\left(L_{\varphi X} \beta-L_{X}\left(\varphi^{*} \beta\right)\right) F=\beta\left(N_{\varphi}(\varphi X, F)\right)$.

By definitions, the condition (37) and the fact that $\varphi(F)=0$

$$
\begin{aligned}
& N_{\varphi}(X, F)=\varphi^{2} \llbracket X, F \rrbracket-\varphi \llbracket \varphi X, F \rrbracket \\
= & \varphi\left(-\varphi\left(L_{F} X\right)+L_{F}(\varphi X)\right)=\varphi\left(\left(L_{F} \varphi\right) X\right)=0 .
\end{aligned}
$$

It follows that $\llbracket X-i \varphi X, \beta+i \varphi^{*} \beta \rrbracket$ is a section of $\operatorname{ker} \eta \oplus \operatorname{ker} F$. Finally, the section $\llbracket X-i \varphi X, \beta+i \varphi^{*} \beta \rrbracket$ is in $E^{(1,0)}$ if and only if its $E^{(0,1)}$-part vanishes. If one evaluates the $E^{(0,1)}$-part of $\llbracket X-i \varphi X, \beta+i \varphi^{*} \beta \rrbracket$ on a section $Y$ of ker $\eta$, it is equal to

$$
\beta\left(N_{\varphi}(X, Y)\right)-i \beta\left(N_{\varphi}(\varphi X, Y)\right) .
$$

It vanishes due to (37) and the definition of $\beta$. Therefore, the integrability does not impose any constraint beyond (37).

Corollary 5.4. The generalized almost contact structure associated to a normal contact structure $(\varphi, F, \eta)$ is integrable.

Proof: A normal contact structure in the classical sense satisfies the following conditions. $N_{\varphi}=-F \otimes d \eta, L_{F} \varphi=0$, and $L_{F} \eta=0$. Note that given the other conditions, the identity $N_{\varphi}(X, F)=-d \eta(X, F) F$ is equivalent to $L_{F} \eta=0$. 
5.3. Alternative approach using Courant algebroids. As noted in Section 3.4 , an alternative and more general notion of generalized almost contact structures is a certain bundle map $\widetilde{I}$ on $E^{1}(M)$. In this context, one may consider the integrability of $\widetilde{I}$ in terms of the closure of its $\pm i$-eigenbundles with respect to an extended Courant bracket on $E^{1}(M)$ [24]. We shall address the integrability in this sense as " $E^{1}(M)$-integrability".

It is also noted that if one subjects an almost contact structure to integrability in this sense, then it is again a normal contact structure. If one subjects an almost cosymplectic structure $(\eta, \theta)$ to integrability in this sense, then $\theta=d \eta$, meaning that it is a contact structure [12].

\section{Discussion}

Our review on classical geometry in Section 2 indicates that almost cosymplectic structures is a natural and direct generalization of contact structures. On the other hand, the notion of almost contact structures provides an obvious analogue to define generalized almost contact structures. Although the generalization is straightforward, it is broad enough to include both almost contact structures and almost cosymplectic structures as natural examples. Since our definition of generalized almost contact structures is defined solely by tensorial objects, we propose to use it to replace a broader definition given in [12].

To consider integrability of generalized almost contact structures, we compare Proposition 4.4 and Proposition 5.3 here and Theorem 5.3 in [12] to find that the notion of integrability on the cone and $E^{1}(M)$-integrability for almost contact structures leads to normal contact structures. The Courant-integrability leads to a technical and slightly weaker requirement.

As demonstrated by Proposition 4.3 and Proposition 5.4 in [12], both integrability on the cone and $E^{1}(M)$-integrability of an almost cosymplectic structure $(\eta, \theta)$ imply that it is a contact structure. i.e., $\theta=d \eta$ However, Proposition 5.2 shows that Courant-integrability only requires $d \theta=0$. In particular, the generalized almost contact structures associated to classical contact structures are Courant-integrable. It demonstrates that a Sasakian approach to integrability does not accommodate cosymplectic structures although cosymplectic structures are integrable G-structures. 
The relatively weak requirement due to the Courant-integrability interests us as it allows us to control the geometry as well as to maintain meaningful flexibility.

A feature of the theory of generalized complex structures is a deformation theory allowing extrapolation between complex and symplectic structures [9] [17]. It is in sharp contrast to the well known fact that deformation of symplectic structures on compact manifolds are trivial [16].

Deformation of contact structures on compact manifolds is also known to be trivial [10]. A natural question now is to what extent one could replicate the theory of generalized complex structures to develop a non-trivial deformation theory for generalized contact structures. Results in Proposition 4.3 and Proposition 5.2 indicate that Courant-integrability provides a relative large class of structures for analysis. Given Definition 3.3, we expect a deformation theory to allow a contact structure, as an almost cosymplectic structure, to deform to an almost contact structure. We will develop such a deformation in a forthcoming paper [18].

Acknowledgement. We thank the referee for helpful suggestions.

\section{REFERENCES}

[1] D. E. Blair, Riemannian Geometry of Contact and Symplectic Manifolds, Progress in Mathematics 203, Birkhäuser, 2001.

[2] C. P. Boyer \& K. Galicki, Sasakian Geometry, Oxford University Press, Oxford, 2008.

[3] S. S. Chern, Pseudo-groupes continus infinis, Colloques internationaux du C.N.R.S., Strasbourg, (1953) 119-136.

[4] S. S. Chern, The geometry of G-structures, Bull. Amer Math. Soc. 72 (1966) 167-219.

[5] M. Crainic, Generalized complex structures and Lie brackets, preprint arXiv:math/0412097.

[6] T. Courant, Dirac structures, Trans. Amer. Math. Soc. 319 (1990), 631-661.

[7] T. Courant \& A. Weinstein, Beyond Poisson structures, in Séminaire Sud Rhodanien de Geométrie, Travaux en cours 2 Hermann, Paris, (1988) 39-49.

[8] A. Fujimoto, Theory of G-structures, English Translation, Publications of the Study Group of Geometry, Volume 1, Okayama University, Okayama, Japan, 1972.

[9] M. Gualtieri, Generalized complex geometry, Ph.D. thesis. Oxford. (2003). arXiv:math.DG/0401221.

[10] J. W. Gray, Some global properties of contact structures, Ann. Math. 69 (1959) 421-450.

[11] N. Hitchin, Generalized Calabi-Yau manifolds, Quart. J. Math. 54 (2003) 281-308.

[12] D. Iglesias-Ponte \& A. Wade, Contact manifolds and generalized complex structures, J. Geom. Phys. 53 (2005) 249-258. 
[13] S. Kobayashi, Transformation Groups in Differential Geometry, Ergebnisse der Mathematik und ihrer Grenzgebiete, 70 Springer-Verlag, New York, 1972.

[14] P. Libermann, Sur les automorphismes infinitésimaux des structures symplectiques et de structures de contact, Coll. Géom. Diff. Globale (Bruxelles 1958), Gauthier-Villars, Paris, 37-59.

[15] S. Lie, Theorie der Transformationgsgruppen, 2 Abschnitt, Teubner, Leipzig, 1890.

[16] J. Moser, On the volume element on a manifold, Trans. Amer. Math. Soc. 120 (1965) 286-294.

[17] Y. S. Poon, Extended deformation of Kodaira surfaces, J. reine angew. Math. 590 (2006), $45-65$.

[18] Y. S. Poon \& A. Wade, Generalized contact structures, manuscript (2009).

[19] S. Sasaki, On differentiable manifolds with certain structures which are closely related to almost contact structure I, Tôhoku Math. J. 12 (1960) 459-476.

[20] S. Sasaki, Almost contact manifolds, Lecture Notes 1, Mathematical Institute, Tôhoku Univeristy (1965).

[21] S. Sasaki \& Y. Hatakeyama, On differentiable manifolds with certain structures which are closely related to almost contact structure II, Tôhoku Math. J 13 (1961) 281-294.

[22] S. Sasaki \& C.-J. Hsu, On the integrability of almost contact structure, Tôhoku Math. J 14 (1962) 167-176.

[23] I. Vaisman, Dirac structures and generalized complex structures on $T M \times \mathbb{R}^{h}$, Adv. Goem. 7 (2007) 453-474.

[24] A. Wade, Conformal Diract structures, Lett. Math. Phys. 53 (2000) 331-348.

\title{
Yat Sun Poon
}

Department of Mathematics

University of California at Riverside, Riverside CA 92521

U.S.A.

Email: ypoon@ucr.edu

\author{
Aïssa Wade \\ Department of Mathematics \\ The Pennsylvania State University, University Park, PA 16802 \\ U.S.A. \\ E-mail: wade@math.psu.edu
}

